\title{
Simplifying Gait Design via Shape Basis Optimization
}

\author{
Chaohui Gong, Daniel I. Goldman and Howie Choset
}

\begin{abstract}
Gaits are crucial to the performance of locomotors. However, it is often difficult to design effective gaits for complex locomotors. Geometric mechanics offers powerful gait design tools, but the utilities of these tools have been limited to systems with two joints. Using shape basis functions, it is possible to approximate the kinematics of complex locomotors using only two shape variables. As a result, the tools of geometric mechanics can be used to study complex locomotion in an intuitive way. The choice of shape basis functions plays an important role in determining gait kinematics, and therefore the performance of a locomotor. To find appropriate basis functions, we introduce the shape basis optimization algorithm, an algorithm that iteratively improve basis functions to find effective kinematic programs. Applying this algorithm to a snake robot resulted a novel gait, which improves its speed of swimming in granular materials.
\end{abstract}

\section{INTRODUCTION}

Geometric mechanics [1]-[4] offers a powerful framework for studying a wide range of locomotor behaviors [5]-[8]. Comparing to conventional planning/design techniques, geometric mechanics offers a set of tools, including connection vector fields and height functions [9], [10], for intuitively analyzing and designing locomotor kinematics.

Past uses of the gait design tools of geometric mechanics were mainly limited to systems which only had two internal degrees of freedom (DoFs) [9]-[13]. We show the shapes of systems that have many DoFs can be approximated as a linear combination of two shape basis functions. These shape basis functions can be incorporated into the formulation of geometric mechanics and, as a result, the tools of geometric mechanics can be applied to design gaits for complex systems. We demonstrate this technique on a snake robot, a serial chain of degrees of freedom which are coordinated to produce locomotion.

It is of critical important to choose proper shape basis functions so that effective kinematic programs can be found. In the past, shape basis functions were either derived from observations on biological systems [14], [15] or determined empirically from robot experiments [16], [17]. Instead, this paper proposes an analytical approach, called the shape basis optimization algorithm which iteratively improves the choice of shape basis functions. Applying the shape basis optimization algorithm to a snake robot resulted in a novel gait which significantly improved its speed of swimming in granular materials.

\section{Geometric Mechanics}

This work draws heavily on the tools of geometric mechanics and necessitating a brief overview of such techniques. For a more comprehensive review of geometric mechanics, see [1]-[4]. For a class of mechanical systems, called principally

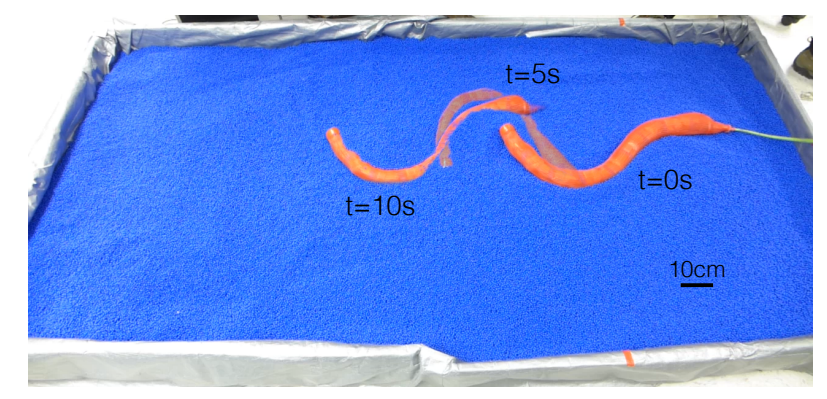

Fig. 1: A snake robot executing the optimized gait. Time interval between each snapshot is five seconds.

kinematic systems, their equations of motion can be expressed as [6], [7]

$$
\boldsymbol{\xi}=\mathbf{A}(\boldsymbol{\alpha}) \dot{\boldsymbol{\alpha}},
$$

where $\boldsymbol{\xi}$ denotes the body velocity and $\boldsymbol{\alpha}$ denotes the joint angles. In kinematic systems, inertial effects are negligible. They do not coast and will stop immediately when joints are frozen.

Equation (1) is referred to as the kinematic reconstruction equation, and $\mathbf{A}(\boldsymbol{\alpha})$ is called the local connection, a matrix that relates shape velocity $\dot{\alpha}$ to body velocity $\boldsymbol{\xi}$. The kinematic reconstruction equation separates group variables (position and orientation) from shape variables (joint angles), and builds an explicit mapping from internal shape changes to displacements in the workspace. By analyzing this mapping, one can readily design gaits for locomoting systems, and we will exploit the structure of this mapping to optimize gaits.

\section{A. Connection Vector Fields and Height Function}

For a planar system with only two joints, i.e. $\alpha \in \mathbb{R}^{2}$, the local connection $\mathbf{A}(\boldsymbol{\alpha})$ is a $3 \times 2$ matrix. The body velocities in the $x, y$ and $\theta$ directions respectively are computed as the dot product between each row of $\mathbf{A}(\boldsymbol{\alpha})$ and $\dot{\boldsymbol{\alpha}}$. Each row of $\mathbf{A}(\boldsymbol{\alpha})$ can be visualized as a connection vector field (see Figure 2), which graphically shows the kinematics of the system. Intuitively speaking, a shape velocity $\dot{\alpha}$ in the direction of the vector field would produce the largest possible body velocity in the specific direction, whereas a shape velocity $\dot{\alpha}$ perpendicular to the vector field would yield zero body velocity.

A gait, i.e. cyclic shape change, is represented as a closed curve in the shape space. The displacement resulting from a gait $\partial \phi$ can be approximated by the line integral

$$
\left[\begin{array}{c}
\Delta x \\
\Delta y \\
\Delta \theta
\end{array}\right] \approx \int_{\partial \phi} \mathbf{A}(\boldsymbol{\alpha}) \mathrm{d} \boldsymbol{\alpha} .
$$

According to Stokes' Theorem, the line integral along a closed curve $\partial \phi$ is equal to the area integral of the curl of $\mathbf{A}(\alpha)$ over 

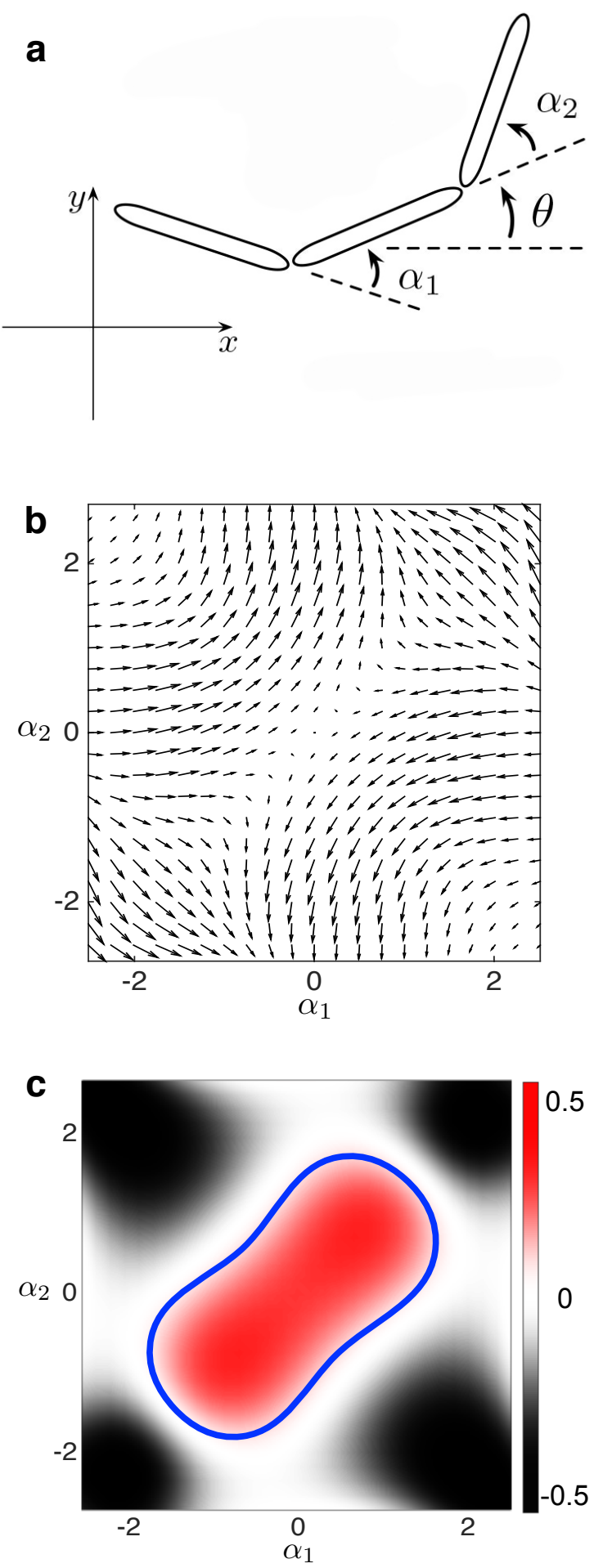

Fig. 2: The connection vector field and height function corresponding to the forward motion of a three-link swimmer in a viscous fluid. (a) A three-link swimmer. (b) The connection vector field corresponding to motions in the forward direction. (c) The height function corresponding to motions in the forward direction the surface area enclosed by $\partial \phi$,

$$
\int_{\partial \phi} \boldsymbol{A}(\boldsymbol{\alpha}) \mathrm{d} \boldsymbol{\alpha}=\iint_{\phi} \nabla \times(\mathbf{A}(\boldsymbol{\alpha})) \mathrm{d} \alpha_{1} \mathrm{~d} \alpha_{2},
$$

where $\phi$ denotes the surface bounded by $\partial \phi$. The curl of the connection vector field $\nabla \times(\mathbf{A}(\boldsymbol{\alpha}))$ is referred to as the height function [10].

Determining a gait that produces the largest displacement per cycle can be quite challenging with the connection vector fields. However, with the height function a gait that can produce the largest displacement per cycle can be determined by finding the zero set which has the largest area integral. For example, Figure 2 shows the connection vector field and height function corresponding to the forward direction of a three-link swimmer in a viscous fluid [18]. Given the structure of the height function, the gait that can produce the largest displacement can be identified as the zero-set of the height function, which is highlighted as the blue curve.

The height function represents a distinct class of gait optimization technique which does not require parameterizations of stroke patterns. In comparison, Tam and Hosoi [19] successfully determined the optimal stroke of a three-link swimmer by modeling joint trajectories as Fourier series. Kanso [20] empirically found the optimal undulatory pattern of a continuum swimmer assuming a sinusoidal body wave.

\section{B. Average Body Frame}

In general, the integral of body velocity does not equal to actual displacement in the workspace because of the noncommutativity effect of the group space [9]. However, it has been shown that with a properly chosen body frame, called the minimum perturbation coordinates, the integral of body velocity can very well approximate the actual displacements [9]. In this work, we used the average body frame as the frame of reference. The average body frame mitigates the non-commutativity effects in a way similar to the minimum perturbation coordination, and it is relatively easy to compute.

For a planar snake robot with $N$ joints, let $x_{0 l}, y_{0 l}, \theta_{0 l}$ represent the position and orientation of the $l$-th link with respect to the frame of the head (link 0). The position and orientation of the average body frame with respect to the head frame are computed as the average position and orientation of all links,

$$
\begin{aligned}
& \bar{x}=\frac{1}{N+1} \sum_{l=1}^{N+1} x_{l} \\
& \bar{y}=\frac{1}{N+1} \sum_{l=1}^{N+1} y_{l} \\
& \bar{\theta}=\frac{1}{N+1} \sum_{l=1}^{N+1} \theta_{l} .
\end{aligned}
$$

The configuration of the average body frame with respect to the head frame can also be represented as a homogeneous 
transformation matrix

$$
\overline{\boldsymbol{g}}=\left[\begin{array}{ccc}
\cos (\bar{\theta}) & -\sin (\bar{\theta}) & \bar{x} \\
\sin (\bar{\theta}) & \cos (\bar{\theta}) & \bar{y} \\
0 & 0 & 1
\end{array}\right] .
$$

\section{ShAPE BASIS FUNCTIONS}

The utility of the height function technique is limited to systems that have only two internal DoFs due to the lack of generalization of Equation (3) in high dimensional space. Fortunately, evidence from biology suggest body waves can be employed to manage locomotor complexity [21]. And we show these body waves can be mathematically represented as a linear combination of two shape basis functions, a concept originally proposed by Chirikijian and Burdick [22] for modeling the kinematics of hyper-redundant manipulators [23]. And, as a result, the utility of the height function technique can be extended to study gaits of snake robots with many joints.

\section{A. Local Connection with Respect to Shape Basis Functions}

We modeled the shape of a snake robot with $N$ joints as

$$
\boldsymbol{\alpha}=w_{1} \boldsymbol{\beta}_{1}+w_{2} \boldsymbol{\beta}_{2}
$$

where $\boldsymbol{\alpha} \in \mathbb{R}^{N}$ denotes the joint angles and $\boldsymbol{\beta}_{1}, \boldsymbol{\beta}_{2} \in \mathbb{R}^{N}$ are two shape basis functions, which are mutually orthogonal and of unit-length. Equation (5) reduces the control of a snake robot with $N$ joints to controlling two shape variables $\mathbf{w}=$ $\left[\begin{array}{ll}w_{1} & w_{2}\end{array}\right]^{T} \in \mathbb{R}^{2}$. This reduction allows for deriving a local connection with respect to the reduced shape variables $\boldsymbol{w}$,

$$
\boldsymbol{\xi}=\tilde{\mathbf{A}}(\boldsymbol{w}) \dot{\boldsymbol{w}}
$$

where $\tilde{\mathbf{A}}(\mathbf{w})=\mathbf{A}(\alpha) \boldsymbol{J}_{\alpha}$ is the local connection with respect to the reduced shape variables $\boldsymbol{w}$, and $\boldsymbol{J}_{\boldsymbol{\alpha}}$ is the shape Jacobian,

$$
\boldsymbol{J}_{\boldsymbol{\alpha}}=\frac{\partial \boldsymbol{\alpha}}{\partial \boldsymbol{w}}=\left[\begin{array}{ll}
\boldsymbol{\beta}_{1} & \boldsymbol{\beta}_{2}
\end{array}\right] .
$$

\section{B. Low Reynolds Number Swimmer}

We derive the kinematic reconstruction equation for a $N$ joint snake robot swimming in viscous fluid, which will be used to elucidate how shape basis functions help to simplify gait design for high dimensional systems.

Let $\boldsymbol{\xi}_{0 l}$ denote the body velocity of link $l$ with respect to the head frame, which was computed using the following,

$$
\boldsymbol{\xi}_{0 l}=\boldsymbol{J}_{l}^{b}\left[\begin{array}{lll}
\dot{\alpha_{1}} & \cdots & \dot{\alpha}_{l}
\end{array}\right]^{T},
$$

where $\mathbf{J}_{l}^{b} \in \mathbb{R}^{3 \times l}$ denotes the body Jacobian matrix when viewing link $l$ as the tool frame [24] and $(\cdot)^{T}$ means transpose. To simplify derivations, we rewrote (8) into the following,

$$
\boldsymbol{\xi}_{0 l}=\left[\begin{array}{ll}
\boldsymbol{J}_{l}^{b} & \mathbf{0}_{l}
\end{array}\right] \dot{\boldsymbol{\alpha}}=\boldsymbol{J}_{l} \dot{\boldsymbol{\alpha}}
$$

where $\mathbf{0}_{l} \in \mathbb{R}^{3 \times(N-l)}$ denoted a zero matrix and $\boldsymbol{J}_{l} \in \mathbb{R}^{3 \times N}$ is a linear differential map from joint velocity (shape velocity) $\dot{\boldsymbol{\alpha}}$ to $\boldsymbol{\xi}_{0 l}$. We denote the body velocity of the head with respect to the world frame as $\boldsymbol{\xi}_{w 0}$. The body velocity of individual links with respect to the world frame is then computed using the following [24],

$$
\boldsymbol{\xi}_{w l}=\operatorname{Ad}_{g_{0 l}^{-1}} \boldsymbol{\xi}_{w 0}+\boldsymbol{\xi}_{0 l},
$$

where $\operatorname{Ad}_{g}$ denotes the adjoint operator, which maps body velocity between different frames and $\boldsymbol{g}_{0 l}$ denotes the configuration of the $l$-th frame with respect to the head frame.

The viscous force experienced by link $l$ with respect to its own body frame was thus computed as

$$
\boldsymbol{F}_{l}^{b}=-\boldsymbol{K} \boldsymbol{\xi}_{w l}=-\left[\begin{array}{lll}
k_{x} & & \\
& k_{y} & \\
& & k_{\theta}
\end{array}\right] \boldsymbol{\xi}_{w l},
$$

where $k_{x}=1, k_{y}=2, k_{\theta}=1$ assuming each link is thin and long [18]. All the off-diagonal terms of $\boldsymbol{K}$ are zeros. $\boldsymbol{F}_{l}^{b}$ was then transformed into the head frame using the following [24],

$$
\boldsymbol{F}_{l}=\mathrm{Ad}_{\boldsymbol{g}_{0 l}^{-1}}^{T} \boldsymbol{F}_{l}^{b}
$$

where $F_{l}$ denoted the force applied to link $l$ with respect to the head frame. Because inertial effects are negligible during locomotion in a viscous fluid, the net forces experienced on a system sum to zero [11],

$$
\sum_{i=0}^{N} \boldsymbol{F}_{i}=\mathbf{0}
$$

Rewriting Equation (13) in terms of $\dot{\alpha}$ and $\xi_{w 0}$ led to

$$
\sum_{l=0}^{N}\left(\operatorname{Ad}_{\boldsymbol{g}_{0 l}^{-1}}^{T} \boldsymbol{K}\left(\operatorname{Ad}_{\boldsymbol{g}_{0 l}^{-1}} \boldsymbol{\xi}_{w 0}+\boldsymbol{J}_{l} \dot{\boldsymbol{\alpha}}\right)\right)=\mathbf{0} .
$$

A close inspection to Equation (14) shown that the only unknown variable was the body velocity of the head frame, $\xi_{w 0}$. Rearranging (14) resulted in the following,

$$
\underbrace{\left(\sum_{l=0}^{N}\left(\operatorname{Ad}_{\boldsymbol{g}_{0 l}^{-1}}^{T} \boldsymbol{K} \operatorname{Ad}_{\boldsymbol{g}_{0 l}^{-1}}\right)\right)}_{\boldsymbol{\omega}_{\xi}} \boldsymbol{\xi}_{w 0}=-\underbrace{\sum_{l=0}^{N}\left(\operatorname{Ad}_{\boldsymbol{g}_{0 l}^{-1}}^{T} \boldsymbol{K} \boldsymbol{J}_{l}\right)}_{\boldsymbol{\omega}_{\alpha}} \dot{\boldsymbol{\alpha}} .
$$

The body velocity of the head frame, $\xi_{w 0}$, was then computed as

$$
\boldsymbol{\xi}_{w 0}=-\boldsymbol{\omega}_{\xi}^{-1} \boldsymbol{\omega}_{\alpha} \dot{\boldsymbol{\alpha}}=\mathbf{A}(\boldsymbol{\alpha}) \dot{\boldsymbol{\alpha}},
$$

which was purely determined by shape velocity $\dot{\boldsymbol{\alpha}}$.

To mitigate the non-commutativity effects of group $\mathbf{S E}(2)$ and to intuitively represent the whole body motion of a undulating snake robot, we used the average body frame as the frame of reference of the robot motion. The body velocity of the average body frame with respect to the world can be derived from the body velocity of the head frame as,

$$
\begin{aligned}
\overline{\boldsymbol{\xi}} & =\mathbf{A d}_{\bar{g}} \boldsymbol{\xi}_{w 0}+\overline{\boldsymbol{g}}^{-1} \dot{\overline{\boldsymbol{g}}} \\
& =\operatorname{Ad}_{\bar{g}} \mathbf{A}(\boldsymbol{\alpha}) \dot{\boldsymbol{\alpha}}+\overline{\boldsymbol{g}}^{-1} \frac{\partial \overline{\boldsymbol{g}}}{\partial \boldsymbol{\alpha}} \dot{\boldsymbol{\alpha}},
\end{aligned}
$$

where $\overline{\boldsymbol{g}}$ denotes the average body frame. Therefore, Equation (15) can be mapped to the average body frame,

$$
\overline{\boldsymbol{\xi}}=\left(\operatorname{Ad}_{\bar{g}} \mathbf{A}(\boldsymbol{\alpha})+\overline{\boldsymbol{g}}^{-1} \frac{\partial \overline{\boldsymbol{g}}}{\partial \boldsymbol{\alpha}}\right) \dot{\boldsymbol{\alpha}}
$$


By incorporating shape basis functions into the above equation using Equation (5), the kinematic reconstruction equation can be expressed in the average body frame,

$$
\overline{\boldsymbol{\xi}}=\overline{\mathbf{A}}(\boldsymbol{w}) \dot{\boldsymbol{w}}
$$

where $\overline{\mathbf{A}}(\boldsymbol{w})=\left(\boldsymbol{A d}_{\bar{g}} \mathbf{A}(\boldsymbol{\alpha})+\overline{\boldsymbol{g}}^{-1} \frac{\partial \bar{g}}{\partial \boldsymbol{\alpha}}\right) \boldsymbol{J}_{\boldsymbol{\alpha}}$. Note $\overline{\mathbf{A}}(\boldsymbol{w})$ is a 3 by 2 matrix, which allows us to efficiently design gait using the height function technique.

\section{Shape BASIS Optimization}

The choice of shape basis functions $\boldsymbol{\beta}$ affects gait kinematics (joint trajectories), and therefore the performance of a locomotor. It is crucial to choose appropriate shape basis functions so that effective kinematic program can be found. This section introduces the shape basis optimization algorithm, which is based on gradient ascent, to choose proper shape basis functions.

\section{A. The Serpenoid Curve and Parameterized Gaits}

The serpenoid curve [14] is accepted as a good model to describe the time varying shape of an undulating snake. The serpenoid curve models the body curvature of an undulating snake as a sinusoidal function,

$$
\kappa(s, t)=A \sin (\Omega s+\omega t),
$$

where $s$ denotes the arc length along the body, $A$ denotes the amplitude of the wave, $\omega$ denotes the temporal frequency and $\Omega$ denotes the spatial frequency.

The serpenoid curve [14] can actually be reformulated as a linear combination of two sinusoidal shape basis functions. The spatial and temporal components of a serpenoid curve can be separated as

$$
\begin{aligned}
\kappa(s, t) & =A \sin (\Omega s+\omega t) \\
& =A \cos (\omega t) \sin (\Omega s)+A \sin (\omega t) \cos (\Omega s) .
\end{aligned}
$$

The two spatial-related components $\sin (\Omega s)$ and $\cos (\Omega s)$ can be interpreted as two shape basis functions with $A \cos (\omega t)$ and $A \sin (\omega t)$ as the time varying weights associated with the two shape basis functions. In other words, $w_{1}(t)=A \cos (\omega t)$ and $w_{2}(t)=A \sin (\omega t)$. The weights of the two shape basis functions $\left(w_{1}, w_{2}\right)$ traces a circular path in the two dimensional shape space. The radius of this circle is equal to the wave amplitude $A$ of the serpenoid curve. Therefore, a serpenoid curve can be represented as a circle centered in the middle of the shape space spanned by the two shape basis functions $\sin (\Omega s)$ and $\cos (\Omega s)$.

For gaits with a given parametric form, e.g. the serpenoid curve, gait design was achieved through systematic simulation or experiments with different gait parameters. For example, Sharpe et al. [25] studied how the parameters of a serpenoid curve affect the speed of swimming underneath sand through systematic simulations. Their study revealed biological organisms operate at the wave parameters that maximizes speed of swimming. The compound serpenoid curve [16] is a generalization to the serpenoid curve. It models snake gaits as two body waves in the dorsal and lateral planes. Gait parameters that can achieve useful motions were determined by direct experimentations on hardware [17], [26]. In the central pattern generator (CPG) paradigm [27], [28], joint trajectories are modeled as coupled harmonic oscillators. Gait design in the CPG framework is empirically achieved through systematic testing on different parameters that govern the states of the oscillators. However, with no assumption on the parametric form of joint trajectories, it is challenging to design gaits. The shape basis optimization algorithm provides a non-parametric way to design effective gaits.

\section{B. Optimizing Shape Basis Functions}

To illustrate the principle of the shape basis optimization algorithm we use a four link swimmer in Low Reynolds fluid as an example. A four link swimmer has a threedimensional shape space $\boldsymbol{\alpha}=\left[\begin{array}{lll}\alpha_{1} & \alpha_{2} & \alpha_{3}\end{array}\right]^{T}$, where $\alpha_{1}, \alpha_{2}$ and $\alpha_{3}$ denote the three joint angles. Although the kinematic reconstruction equation of a four link swimmer can be easily derived using the method described in the previous section, it is challenging to design gaits in a computationally efficient and intuitive manner. We hence choose to reduce the three dimensional shape space to a two dimensional one using shape basis functions so that the height function technique can be applied. And, we used the shape basis optimization algorithm to select proper shape basis functions.

The objective of the shape basis optimization algorithm is to choose proper shape basis functions $\boldsymbol{\beta}_{1}, \boldsymbol{\beta}_{2}$ so that the swimmer can achieve maximum forward displacement per gait cycle (using only two basis functions). However, it is not immediately clear $\boldsymbol{\beta}_{1}$ and $\boldsymbol{\beta}_{2}$ would lead to effective movement. We arbitrarily initialized $\boldsymbol{\beta}_{1}^{0}=\left[\begin{array}{lll}1 & 0 & 0\end{array}\right]^{T}$ and $\boldsymbol{\beta}_{2}^{0}=\left[\begin{array}{lll}0 & 0 & 1\end{array}\right]^{T}$. Here the superscript denotes the number of iteration. This particular choice of shape basis functions only use the first and third joints of the swimmer which define a reduced shape space corresponding to the plane spanned by $\alpha_{1}$ and $\alpha_{3}$, as shown in Figure 3. Physical intuition suggests that $\boldsymbol{\beta}_{1}^{0}$ and $\boldsymbol{\beta}_{2}^{0}$ are not good choices for shape basis functions because undulatory motions that use only part of the body are not likely to generate the most effective movements. However, this choice of initial conditions will highlight how the shape basis optimization algorithm can improve from even poor initial choices of shape basis functions.

Given the initial shape basis functions $\boldsymbol{\beta}_{1}^{0}$ and $\boldsymbol{\beta}_{2}^{0}$, we can use the height function technique to identify the optimal gait $\partial \phi^{0}$ in the reduced shape space, shown as the blue curve in Figure 3. With $\partial \phi^{0}$, it is straightforward to compute the forward displacement using

$$
\Delta x^{0}=\int_{\partial \phi} \overline{\mathbf{A}}_{x}^{0}(\boldsymbol{w}) \mathrm{d} \boldsymbol{w},
$$

where $\overline{\mathbf{A}}_{x}^{0}(\boldsymbol{w})$ denotes the first row of $\overline{\mathbf{A}}^{0}(\boldsymbol{w})$, and $\Delta x^{0}$ is the maximum displacement per gait cycle that can possibly be achieved in the two dimensional shape space spanned by $\boldsymbol{\beta}_{1}^{0}$ and $\boldsymbol{\beta}_{2}^{0}$. 

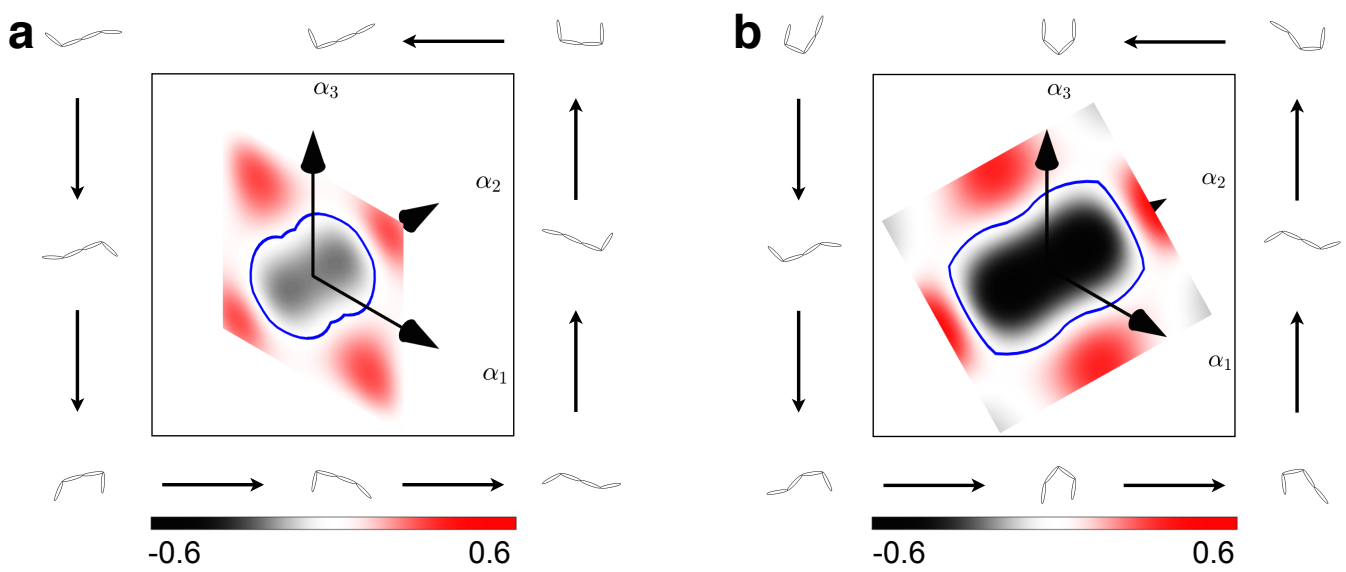

Fig. 3: Height functions of a four link swimmer in viscous fluid. (a) Height function before shape basis optimization. (b) Height function after shape basis optimization. The blue curves represent the optimal gaits in the corresponding reduced shape space. The insets show the evolution of body shape of a four link swimmer while executing the optimal gaits (in two different reduced shape space).

To improve the speed of locomotion, the shape basis functions have to change. The next step is to compute the gradient of $\Delta x^{0}$ with respect to the coefficients of $\boldsymbol{\beta}_{1}^{0}$ and $\boldsymbol{\beta}_{2}^{0}$, and then update the shape basis functions along the direction of the gradient as

$$
\boldsymbol{\beta}_{i}^{t+1}=\boldsymbol{\beta}_{i}^{t}+\eta \frac{\Delta x^{t}}{\boldsymbol{\beta}_{i}^{t}}
$$

where $\beta_{i}^{t}$ denotes a shape basis function in the $t$-th iteration. The gradient provides a direction along which updating the shape basis functions would result in larger displacement per gait cycle.

After each iteration, the algorithm normalizes $\boldsymbol{\beta}_{1}^{t+1}$ and $\boldsymbol{\beta}_{2}^{t+1}$ and then uses the Gram-Schmidt algorithm to force $\boldsymbol{\beta}_{1}^{t+1}$ and $\boldsymbol{\beta}_{2}^{t+1}$ are orthonormal. The entire process repeats until $\boldsymbol{\beta}_{1}^{t}$ and $\boldsymbol{\beta}_{2}^{t}$ (and $\Delta x^{t}$ ) converged. A detailed description of the shape basis optimization algorithm is included in Algorithm 1.

The algorithm converged to two optimal shape basis functions,

$$
\begin{aligned}
& \boldsymbol{\beta}_{1}^{*}=\left[\begin{array}{lll}
-0.73 & 0.04 & 0.69
\end{array}\right]^{T} \\
& \boldsymbol{\beta}_{2}^{*}=\left[\begin{array}{lll}
0.44 & 0.79 & 0.43
\end{array}\right]^{T}
\end{aligned}
$$

In simulation, displacement per cycle of the four link swimmer was improved from 0.06 body length (BL) per cycle to 0.14 BL/cycle.

The process of shape basis optimization can be graphically shown as in Figure 3. When the shape basis functions $\boldsymbol{\beta}_{1}^{t}$ and $\boldsymbol{\beta}_{2}^{t}$ were updated, the plane that represented the reduced shape space spanned by $\boldsymbol{\beta}_{1}^{t}$ and $\boldsymbol{\beta}_{2}^{t}$ rotated in the three-dimensional shape space. While the plane was rotating, the optimal gait in the corresponding reduced shape space changes. When the plane (reduced shape space) reaches a stationary orientation, the optimal gait in this reduced shape space achieved maximum displacement per gait cycle (local optimum). And, the improvement in displacement per gait cycle can be seen from the increased color intensity of the area enclosed by the optimal gait.

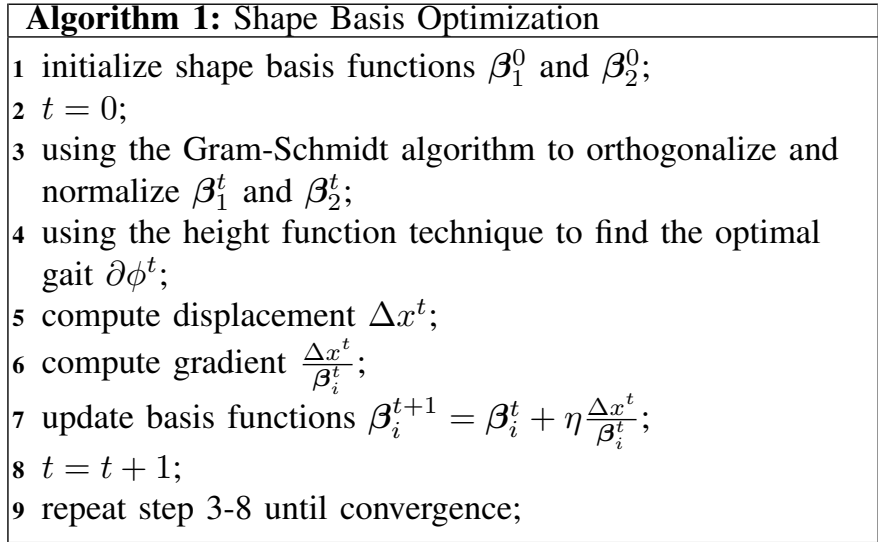

\section{LOCOMOTION IN GRANULAR MATERIALS}

The previous section describes the shape basis optimization algorithm for systems whose kinematic reconstruction equations can be analytically derived. For some types of locomotion, especially for those locomoting in complex substrates, e.g. sand, it is difficult to derive the kinematic reconstruction equation analytically. Alternatively the kinematic reconstruction equation and connection vector fields can be computed numerically [11] for those systems.

\section{A. Granular Resistive Force Theory}

Granular resistive force theory (RFT) [29] has been developed to model the movements of animals and robots within granular media. Prior works [25], [30] have shown simulations using granular RFT agreed well with actual robot and animal movements. We therefore used granular RFT to numerically derive local connections. Doing so allows us to not only design gaits, but also to test these gaits on a robot in a granular material. It is also interesting to examine if granular RFT would serve a reasonable model for studying movements of a robot which is only partially submerged in granular materials.

In granular RFT, the resistive force experienced on an 
infinitesimally small segment of a moving intruder is decomposed into thrust and drag components. The reaction force applied on the entire system is computed as,

$$
\boldsymbol{F}=\int\left(\mathrm{d} \boldsymbol{F}_{\perp}+\mathrm{d} \boldsymbol{F}_{\|}\right)
$$

where $\boldsymbol{F}_{\|}$and $\boldsymbol{F}_{\perp}$ respectively denote forces parallel and perpendicular to a segment. $\boldsymbol{F}_{\perp}$ and $\boldsymbol{F}_{\|}$are only functions of attack angle and are independent from the magnitude of the speed. The attack angle of a moving segment can be computed from its body velocity. Further details regarding granular RFT can be found in [29].

We assumed the motion of a undulating snake in granular material is quasi-static, which means the total net force applied on the system goes to zero

$$
\boldsymbol{F}=\int\left(\mathrm{d} \boldsymbol{F}_{\perp}+\mathrm{d} \boldsymbol{F}_{\|}\right)=0 .
$$

The only unknown in the above equation is the body velocity of the chosen body frame, i.e. the average body frame. Given a shape velocity $\dot{\alpha}$, the body velocity of the average body frame can be computed by solving for Equation (27). In our implementation, we used the MATLAB function fsolve to compute the root of Equation (27).

\section{B. Numerical Computation of the Local Connection}

With given shape basis functions, the local connection of a snake robot moving in granular media can be computed numerically. We uniformly sampled the two dimensional shape space on a 31 by 31 grid where $w_{1}, w_{2} \in[-\pi, \pi]$. At every point $\left(w_{1}, w_{2}\right)$ on the grid, we first locally perturbed the value of the first shape variable $w_{1}:=w_{1}+\epsilon$, while holding the other constant. In practice, the magnitude of perturbation $\epsilon=0.05$. This small change in shape produces a small displacement $\Delta x_{1}^{b}, \Delta y_{1}^{b}$ and $\Delta \theta_{1}^{b}$ measured in the body frame, which can be easily computed using the RFT calculation. Next, we kept the value of the first shape variable constant, but perturbed value of the second shape variable $w_{2}:=w_{2}+\epsilon$. The resultant displacement measured in the body frame were $\Delta x_{2}^{b}, \Delta y_{2}^{b}$ and $\Delta \theta_{2}^{b}$. These numerically calculated displacements served an approximation to the local connection at shape $\left(w_{1}, w_{2}\right)$,

$$
\mathbf{A}\left(w_{1}, w_{2}\right) \approx \frac{1}{\epsilon}\left[\begin{array}{ll}
\Delta x_{1}^{b} & \Delta x_{2}^{b} \\
\Delta y_{1}^{b} & \Delta y_{2}^{b} \\
\Delta \theta_{1}^{b} & \Delta \theta_{2}^{b}
\end{array}\right] .
$$

The same procedure was repeated at every sampled point. This numerically determined local connection can then be used to plot connection vector fields and height functions.

\section{RESUlTS}

\section{A. System}

We tested the efficacy of the shape basis optimization algorithm on a snake robot swimming in a $1 \mathrm{~m} \times 2 \mathrm{~m}$ test bed filled with $6 \mathrm{~mm}$ plastic balls (a type of granular material commonly used in robot experiments [11], [31]) as shown in Figure 1. Four Opti-Track IR 13 cameras located above the
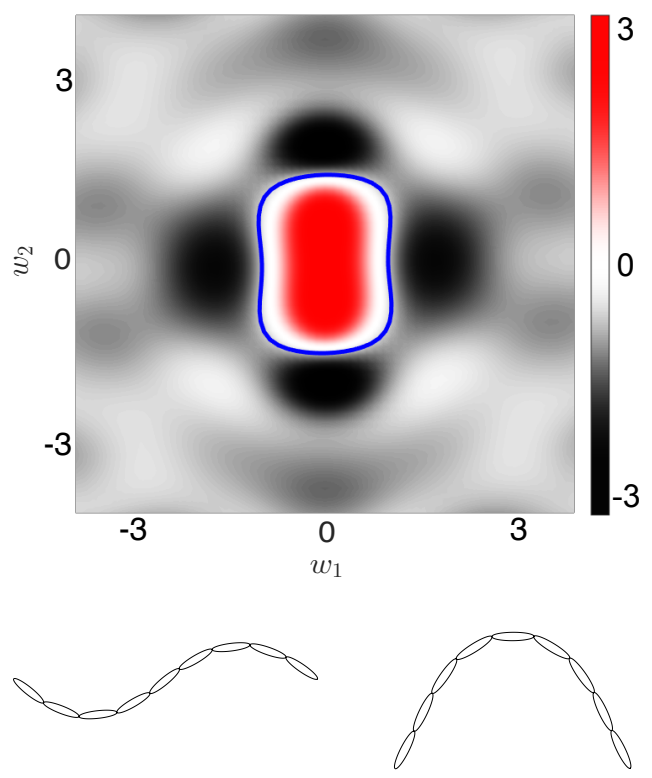

Fig. 4: The two optimized shape basis functions $\boldsymbol{\beta}_{1}^{*}, \boldsymbol{\beta}_{2}^{*}$ and the corresponding optimal gait in the reduced shape space. The blue curve in the top figure shows the optimal gait $\partial \phi^{*}$. The two figures at the bottom show the shape of a snake robot when the two optimized shape basis functions are commanded.

bed were used to record the motion of the robot. The snake robot has 16 joints, which alternatively rotate in the pitch (vertical) and yaw (horizontal) directions. The body length of the robot is $86 \mathrm{~cm}$. Because the robot was only used to generate planar movements, only the eight horizontal joints were used in the experiments. During the experiments, the robot was mostly buried in the material (immersion depth from the bottom surface of the robot to the surface of the granular materials was nearly equal to the diameter of the robot).

\section{B. Shape Basis Optimization}

The serpenoid curve (sinusoidal shape basis functions) has been proved a good model for modeling undulatory locomotion [14]. We therefore initialized the shape basis functions $\boldsymbol{\beta}_{1}^{0}$ and $\boldsymbol{\beta}_{2}^{0}$ as

$$
\begin{aligned}
& \boldsymbol{\beta}_{1}^{0}=\left[\begin{array}{llll}
\sin \left(2 \pi \frac{\Omega}{N}\right) & \sin \left(2 \pi \frac{2 \Omega}{N}\right) & \cdots & \sin \left(2 \pi \frac{N \Omega}{N}\right)
\end{array}\right]^{T} \\
& \boldsymbol{\beta}_{2}^{0}=\left[\begin{array}{llll}
\cos \left(2 \pi \frac{\Omega}{N}\right) & \cos \left(2 \pi \frac{2 \Omega}{N}\right) & \cdots & \cos \left(2 \pi \frac{N \Omega}{N}\right)
\end{array}\right]^{T}
\end{aligned}
$$

where $N=8$ denotes the number of horizontal joints of the snake robot. We chose the spatial frequency $\Omega=1$ as an initial guess, and relied on the shape basis optimization algorithm to determine shape basis functions that can lead to gaits with larger displacement per cycle.

The shape basis optimization algorithm yielded two optimized shape basis functions

$$
\begin{aligned}
& \boldsymbol{\beta}_{1}^{*}=\left[\begin{array}{llll}
0.3049 & 0.4799 & 0.4196 & 0.1619
\end{array}\right. \\
& \left.\begin{array}{llll}
-0.1569 & -0.4081 & -0.4578 & -0.2716
\end{array}\right]^{T} \\
& \boldsymbol{\beta}_{2}^{*}=\left[\begin{array}{lllll}
0.0235 & -0.1661 & -0.3991 & -0.5532
\end{array}\right. \\
& -0.5561 \quad-0.4073 \quad-0.1763 \quad 0.0168]^{T},
\end{aligned}
$$

and the corresponding optimal gait $\partial \phi^{*}$ in the reduced shape space, shown as the blue curve in Figure 4. The insets of 

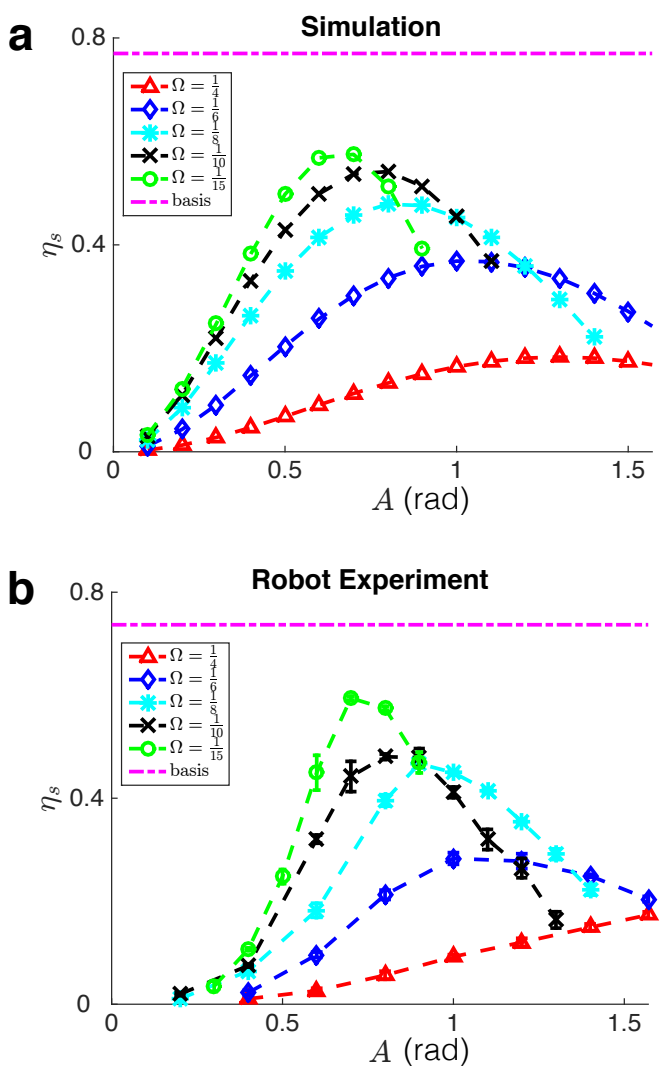

Fig. 5: Displacement per cycle of a snake robot using different gaits in simulation and robot experiments. Undulation efficiency $\eta_{s}$ means displacement per gait cycle normalized by body length. Each marker represents the mean value at a particular value of $\Omega$ and $A$. (a) Comparison of $\eta_{s}$ using different gaits in simulation. (b) Comparison of $\eta_{s}$ using different gaits in robot experiments. The purple line highlights the performance of the optimized gait with standard deviation $=0.007 \mathrm{BL} /$ cycle)

Figure 4 show the resultant body shapes on a snake robot when $\boldsymbol{\beta}_{1}^{*}$ and $\boldsymbol{\beta}_{2}^{*}$ were commanded.

The resultant body shapes generate undulatory patterns similar to that of a serpenoid curve, but differ in some respects. The body shape resulting from the first shape basis function was similar to a serpenoid curve with spacial frequency approximately equal to one (one complete wave along the body). The body shape resulting from the second shape basis function generated half of a wave along the body, and there was a DC component in the joint angles (all the joints bend towards one direction rather than presenting a pure undulatory pattern).

\section{Simulation and Experiment Results}

We next compared displacement per cycle normalized by body length $\eta_{s}$ of the optimized gait $\partial \phi^{*}$ with that of serpenoid curves using different wave parameters in both simulation and robot experiments. To generate serpenoid curves on the snake robot, we used the discrete counterpart to the continuous serpenoid curve in Equation (19),

$$
\alpha(n, t)=A \sin (2 \pi \Omega n+\omega t)
$$

where $A$ denotes the amplitude of the serpenoid curve, $\omega$ denotes the temporal frequency and $\Omega$ denotes the spatial frequency.

In simulation, we sampled amplitudes $A$ from 0.1 to $1.6 \mathrm{rad}$ with an interval $0.1 \mathrm{rad}$, and $\Omega=\frac{1}{15}, \frac{1}{10}, \frac{1}{8}, \frac{1}{6}, \frac{1}{4}$ excluding those combinations of parameters that may cause selfcollision. Displacements per cycle using different wave parameters were calculated using RFT simulation. The simulation results are shown in Figure 5(a).

In robot experiments, we sampled amplitudes $A$ from 0.2 to $1.6 \mathrm{rad}$, and $\Omega=\frac{1}{15}, \frac{1}{10}, \frac{1}{8}, \frac{1}{6}, \frac{1}{4}$ excluding those combinations of parameters that may cause self-collision. During the experiments, the value of temporal frequency was fixed at $\omega=\frac{2 \pi}{10} \mathrm{rad} / \mathrm{s}$ to keep the period of a gait constant. Three trials for each combination of $A$ and $\Omega$ were conducted. The experiment results are shown in Figure 5(b).

We observed that in both simulation and experiments for a fixed value of spatial frequency, if the wave amplitude was too small, the robot did not undulate sufficiently to push against the surrounding material and was not able to generate large displacements. However, if the value of wave amplitude was too large, the highly curved body reduced the effective length of each step in the workspace and, as a result, the robot did move far either. For a fixed value of spatial frequency, there existed an optimal value of wave amplitude at which the robot achieved maximum speed of locomotion. Serpenoid curves with a value of the spatial frequency smaller than $\frac{1}{15}$ or larger than $\frac{1}{4}$ did not generate effective movements on the robot. We observed the maximum speed of locomotion $\eta_{s} \approx 0.59$ BL/cycle was achieved at spatial frequency $\Omega=0.53$ and at wave amplitude $A=0.7 \mathrm{rad}$.

When the optimized gait $\partial \phi^{*}$ was used, the robot was able to achieve a speed of locomotion of $\eta_{s}=0.77 \mathrm{BL} /$ cycle in simulation and $\eta_{s} \approx 0.7 \mathrm{BL} /$ cycle in robot experiments, outperforming the performance of the serpenoid curve even with its best parameters. This result demonstrates the efficacy of the shape basis optimization algorithm in finding proper shape basis functions for finding effective gaits for complex robots.

\section{DISCUSSION}

\section{A. Understanding Shape Basis Functions}

The shape basis optimization algorithm can be understood as a means of finding proper coordinations among all joints. Intuitively speaking, only when all joints "cooperate" with each other can effective movements be achieved. Finding proper coordinations among many joints can be computationally difficult, and the shape basis optimization algorithm provide a way to find proper coordinations. Joint coordinations or shape basis functions provide an appropriate intermediate layer between high level motion plan and low level joint control, allowing for simplified control of robot motion via regulating fewer parameters than directly controlling all the joints.

The idea of shape basis functions is closely related to the concept of kinematic synergies [32]-[34], a concept widely used in robotics. Kinematic synergies widely exists in both manipulations and locomotions, and they have been proven 


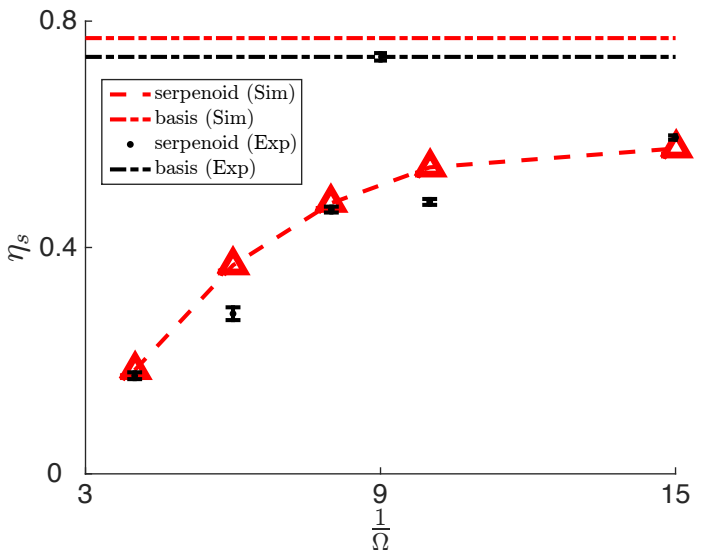

Fig. 6: Comparison between simulation and robot experiments. Red means simulation and black means robot experiments. Each marker represents the maximum displacement per cycle $\eta_{s}$ at a specific value of spatial frequency $\Omega$.

to be an effective strategy to manage complexity in tasks of both locomotion and manipulation. The proposed shape basis optimization algorithm provides a framework to design kinematic synergies.

\section{B. Optimality}

It is worth noting that the optimized gait $\partial \phi^{*}$ only achieved a locally optimal solution. The shape basis optimization algorithm assumed a two-dimensional shape space. While it is possible to find better gaits in a higher dimensional shape space using other expensive computational techniques, by restricting to a two-dimensional shape space the shape basis optimization algorithm balances the performance of gaits and computational efficiency (also making analysis more intuitive using the height function).

The objective of this work is not to find the globally optimal gait, but to demonstrate the importance of finding a proper low-dimensional shape space while designing gaits for complex systems. By representing the complex kinematics of a locomotor using only two shape basis functions, effective gaits can be found in a computationally efficient and intuitive way.

\section{Implications on Biological Systems}

The optimization criterion in the shape basis optimization algorithm was displacement per cycle. However, energetic efficiency might be a more preferable metric to optimize for both biological and robotic systems. Nonetheless, the optimized shape basis functions $\boldsymbol{\beta}_{1}^{*}$ and $\boldsymbol{\beta}_{2}^{*}$ exhibit patterns very similar to the wave patterns displayed on some species of biological snakes, e.g. Chionactis occipitalis. This similarity suggests the waveforms displayed on these biological snakes have the benefit of producing high speed motion. The slight differences between the body waveforms observed on biological snakes and the optimized basis functions are likely due to the differences in optimization criterion, which requires further studies in the future.

\section{Comparing Simulation to Robot Experiments}

Figure 6 shows a comparison between results from simulation and robot experiments. Although we have ignored the surface effects of granular materials in simulation, simulation results agree with robot experiments. The differences between simulation and experiments are partially due to the surface effects of granular materials, which require further investigation in the near future. Another possible source of differences between simulation and experiments is the joint-level control laws of the robot. In the experiments, proportional control was used. As a result, the joint angles might not have perfectly tracked the commanded joint trajectories, causing a difference between simulation and experiments.

\section{E. Initialization of Shape Basis Functions}

Because of the non-linear nature of shape basis optimization for high dimensional locomoting systems, the optimized shape basis functions sensitively depend on the initial values of $\beta_{1}^{0}$ and $\beta_{2}^{0}$. In this work, we used serpenoid curves to initialize shape basis functions. In the future, other forms of basis functions can be considered. It is also possible to use various sampling techniques to start shape basis optimization at different initial values of shape basis functions.

\section{CONCLUSION}

Shape basis functions are useful for simplifying the representation of complex kinematics. By representing the complex kinematics of a high DoFs locomotors with two shape variables, the tools of geometric mechanics can be applied to design gaits in an intuitive manner. The proposed shape basis optimization algorithm is useful to find proper shape basis functions. Applying the shape basis optimization algorithm to a snake robot resulted in a novel gait which improved its speed of swimming in granular materials.

Optimizing shape basis functions can be understood as designing coordinations among many joints. Designing gaits by starting from design coordination among joints builds a novel and general framework for designing gaits for complex locomoting devices. The proposed shape basis optimization algorithm establishes such a framework to first design proper joint coordination, and then design effective gaits in this space. Although we have focused our application on a snake robot, the generality and predictive power of the proposed framework would benefit the analysis of other types of locomotors.

In the future, various types of optimization techniques can be incorporated into the shape basis optimization algorithm for finding good shape basis functions. In addition, this work has focused on the metric of displacement per cycle. In the future, power metrics [35] can be considered to design energetically efficient gaits.

\section{ACKNOWLEDGMENTS}

This research is supported by NSF Grant 1462555 and Robotics Technology Collaborative Alliance. 


\section{REFERENCES}

[1] J. Marsden, Introduction to Mechanics and Symmetry. Springer-Verlag, 1994.

[2] J. E. Marsden, "Geometric foundations of motion and control," in Motion, Control, and Geometry: Proceedings of a Symposium, Board on Mathematical Science, National Research Council Education. The National Academies Press, Conference Proceedings.

[3] A. Shapere and F. Wilczek, "Geometry of self-propulsion at low Reynolds number,' Journal of Fluid Mechanics, vol. 198, pp. 557-585, 1989.

[4] F. Wilczek and A. Shapere, Geometric phases in physics. World Scientific, 1989, vol. 5.

[5] J. Aguilar, T. Zhang, F. Qian, M. Kingsbury, B. McInroe, N. Mazouchova, C. Li, R. Maladen, C. Gong, M. Travers, R. L. Hatton, H. Choset, P. B. Umbanhowar, and D. I. Goldman, "Locomotion robophysics: the study of movement at the intersection of robotics, soft matter and dynamical systems, in review," Reports on Progress in Physics, 2015.

[6] S. Kelly and R. M. Murray, "Geometric Phases and Robotic Locomotion,” J. Robotic Systems, vol. 12, no. 6, pp. 417-431, Jan 1995.

[7] J. Ostrowski and J. Burdick, "The geometric mechanics of undulatory robotic locomotion," International Journal of Robotics Research, vol. 17, no. 7, pp. 683-702, 1998.

[8] R. W. Batterman, "Falling cats, parallel parking, and polarized light," Studies in History and Philosophy of Science Part B: Studies in History and Philosophy of Modern Physics, vol. 34, no. 4, pp. 527-557, 2003.

[9] R. L. Hatton and H. Choset, "Geometric motion planning: The local connection, stokes theorem, and the importance of coordinate choice," The International Journal of Robotics Research, vol. 30, no. 8, pp. 988-1014, 2011. [Online]. Available: http://ijr.sagepub.com/content/30/ 8/988.abstract

[10] E. A. Shammas, H. Choset, and A. A. Rizzi, "Geometric motion planning analysis for two classes of underactuated mechanical systems," Int. J. of Robotics Research, vol. 26, no. 10, pp. 1043-1073, 2007.

[11] R. L. Hatton, Y. Ding, H. Choset, and D. I. Goldman, "Geometric visualization of self-propulsion in a complex medium," Physical review letters, vol. 110, no. 7, p. 078101, 2013.

[12] R. L. Hatton, L. J. Burton, A. E. Hosoi, and H. Choset, "Geometric Maneuverability, with Applications to Low Reynolds Number Swimming," in Proceedigns of the IEEE/RSJ International Conference on Intelligent Robots and Systems, San Francisco, CA USA, September 2011.

[13] E. A. Shammas, H. Choset, and A. A. Rizzi, "Towards a Unified Approach to Motion Planning for Dynamic Underactuated Mechanical Systems with Non-holonomic Constraints," The International Journal of Robotics Research, vol. 26, no. 10, pp. 1075-1124, 2007. [Online]. Available: http://ijr.sagepub.com/cgi/content/abstract/26/10/1075

[14] S. Hirose, Biologically Inspired Robots (Snake-like Locomotor and Manipulator). Oxford University Press, 1993.

[15] H. Marvi, C. Gong, N. Gravish, H. Astley, M. Travers, R. L. Hatton, J. R. Mendelson, H. Choset, D. L. Hu, and D. I. Goldman, "Sidewinding with minimal slip: Snake and robot ascent of sandy slopes," Science, vol. 346, no. 6206, pp. 224-229, 2014.

[16] M. Tesch, K. Lipkin, I. Brown, R. Hatton, A. Peck, J. Rembisz, and H. Choset, "Parameterized and scripted gaits for modular snake robots," Advanced Robotics, vol. 23, no. 9, pp. 1131-1158, 2009.

[17] K. Melo and L. Paez, "Experimental determination of control parameter intervals for repeatable gaits in modular snake robots," in Safety, Security, and Rescue Robotics (SSRR), 2014 IEEE International Symposium on, Oct 2014, pp. 1-7.

[18] R. L. Hatton and H. Choset, "Geometric swimming at low and high reynolds numbers," Robotics, IEEE Transactions on, vol. 29, no. 3, pp. 615-624, 2013.

[19] D. Tam and A. E. Hosoi, "Optimal stroke patterns for Purcell's three-link swimmer," Phys. Review Letters, vol. 98, no. 6, p. 068105, 2007.

[20] E. Kanso, "Swimming Due to Transverse Shape Deformations," Journal of Fluid Mechanics, vol. 631, pp. 127-148, 2009.

[21] H. Astley, C. Gong, M. Travers, M. Serrano, P. Vela, H. Choset J. Mendelson, D. Hu, and D. Goldman, "Modulation of orthogonal body waves enables high maneuverability in sidewinding locomotion," Proceedings of the National Academy of Sciences, 2014.

[22] G. Chirikjian and J. Burdick, "A Modal Approach to Hyper-redundant Manipulator Kinematics," in IEEE Transactions on Robotics and Autmation, vol. 10, 1994, pp. 343-354.
[23] G. Chirikjian and J. Burdick, "Kinematics of Hyper-redundant Locomotion with Applications to Grasping," in IEEE International Conference on Robotics and Autmation, Sacramento, April 1991, pp. 720-727.

[24] R. M. Murray, Z. Li, and S. S. Sastry, A Mathematical Introduction to Robotic Manipulation. CRC Press, 1994.

[25] S. S. Sharpe, S. A. Koehler, R. M. Kuckuk, M. Serrano, P. A. Vela, J. Mendelson, and D. I. Goldman, "Locomotor benefits of being a slender and slick sand swimmer," vol. 218, no. 3, pp. 440-450, 2015.

[26] C. Gong, M. J. Travers, X. Fu, and H. Choset, "Extended gait equation for sidewinding," Robotics and Automation (ICRA), 2013 IEEE International Conference on, 2013.

[27] J. Gonzalez-Gomez, H. Zhang, E. Boemo, and J. Zhang, "Locomotion Capabilities of a Modular Robot with Eight Pitch-Yaw-Connecting Modules," in 9th International Conference on Climbing and Walking Robots., 2006.

[28] A. J. Ijspeert, "Central Pattern Generators for Locomotion Control in Animals and Robotics," Neural Networks, vol. 21, pp. 642-653, 2008.

[29] T. Zhang and D. I. Goldman, "The effectiveness of resistive force theory in granular locomotion," Physics of Fluids, vol. 26, no. 10, 2014. [Online]. Available: http://scitation.aip.org/content/aip/journal/pof2/26/ 10/10.1063/1.4898629

[30] C. Li, T. Zhang, and D. I. Goldman, "A terradynamics of legged locomotion on granular media," Science, vol. 339, no. 6126, pp. 1408-1412, 2013. [Online]. Available: http://science.sciencemag.org/ content/339/6126/1408

[31] R. D. Maladen, Y. Ding, P. B. Umbanhowar, A. Kamor, and D. I. Goldman, "Mechanical models of sandfish locomotion reveal principles of high performance subsurface sand-swimming," Journal of The Royal Society Interface, vol. 8, no. 62, pp. 1332-1345, 2011. [Online]. Available: http://rsif.royalsocietypublishing.org/content/8/62/1332

[32] S. M. Freitas, M. Duarte, and M. L. Latash, "Two kinematic synergies in voluntary whole-body movements during standing," Journal of Neurophysiology, vol. 95, no. 2, pp. 636-645, 2006. [Online]. Available: http://jn.physiology.org/content/95/2/636

[33] H. Hauser, G. Neumann, A. Ijspeert, and W. Maass, "Biologically inspired kinematic synergies provide a new paradigm for balance control of humanoid robots," in Humanoid Robots, 2007 7th IEEE-RAS International Conference on, Nov 2007, pp. 73-80.

[34] S. Vernazza-Martin, N. Martin, and J. Massion, "Kinematic synergies and equilibrium control during trunk movement under loaded and unloaded conditions," Experimental Brain Research, vol. 128, no. 4, pp. 517-526. [Online]. Available: http://dx.doi.org/10.1007/s002210050874

[35] T. Dear, R. Hatton, and H. Choset, "Nonlinear dimensionality reduction for kinematic cartography with an application toward robotic locomotion," in Intelligent Robots and Systems (IROS 2014), 2014 IEEE/RSJ International Conference on, Sept 2014, pp. 3604-3609. 